\title{
The Humanistic Scholars Project: A Study of Attitudes and Behavior Concerning Collection Storage and Technology
}

\section{Wendy P. Lougee, Mark Sandler, and Linda L. Parker}

An assessment of humanist research behaviors and attitudes toward remote storage of collections and technology was conducted at the University of Michigan Library. In an attempt to attenuate negative attitudes toward remote shelving through enhanced bibliographic access, project participants were given direct access to online systems containing records for stored titles. Results suggested this specific intervention was not successful given negative attitudes toward technology. However, attitudes toward the Library grew more positive and increased use of the remote shelving facility was reported. Findings are interpreted in terms of facultylibrarian interactions and faculty acceptance of library programs.

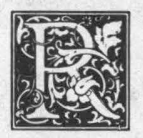

emote storage of library collections is both a long-standing issue and a growing phenomenon. Libraries have long pondered the use of remotely housed collections and how best to select materials for those collections. ${ }^{1}$ During the halcyon days when new construction projects were prevalent, issues of remote storage were less pressing and libraries focused on expanding collections within open stack facilities. But as these boom days waned, libraries once again faced the unpopular decision to develop more economical facilities. While permanent, closed-access facilities for books are not inexpensive, cost studies of storage utilizing a closed-access organization show a sig- nificant savings over the cost of conventional, open-access shelving. ${ }^{2}$

Several studies conducted in the 1970s pointed to the problems experienced by libraries as they implemented collection storage programs. A 1974 study revealed that of 105 libraries, 37 were storing significant portions of their collection and 13 more said that storage was imminent. The same survey suggested few libraries had guidelines for book storage, and few had developed procedures for complete record keeping. ${ }^{3}$ A more recent survey of storage programs of 22 research libraries conducted by the Association for Research Libraries in 1977 reported little uniformity among libraries in terms of criteria for selecting materials for storage, document

Wendy P. Lougee, is Head, Graduate Library and Mark Sandler is Coordinator, Graduate Library Collection, at the University of Michigan, Ann Arbor, Michigan 48109-1205. Linda L. Parker is Chair, Central References Services, at the University of Nebraska, Lincoln, Nebraska 68588-0410. The authors wish to thank project consultants Dr. Margaret Taylor and Dr. Elaine Hochman for their assistance in project design and data analysis. Funding for the project was provided by the Council on Library Resources and the Andrew W. Mellon Foundation. 
delivery, bibliographic access, or preservation. Another point was the distinct lack of information that could be used by a manager in planning for an optimal storage facility. More specifically, there is an absence of reported efforts to analyze user attitudes toward storage. ${ }^{4}$

One common theme precedes the literature on remote storage of collections: remote storage is an unpopular and oftentimes untenable solution for scholars. Storage in a remote facility leads to disagreements on which books should be stored, time lags for delivery, loss of the opportunity to browse the shelves, problems in accurate bibliographic access, and deterioration of books as a result of inadequate environmental controls in the storage facility. The concept of remote storage of collections has often been most vigorously opposed by scholars in the humanities. Perhaps chief among the complaints raised by humanists is the use of book selection criteria based on age or frequency of use. Because the humanities tend to reexamine the classics with every generation, storage selection models based upon use criteria frequently do not appear relevant to humanities disciplines.

Given the nature of research strategies in the humanities, it is quite understandable that humanists should resist remote storage of library collections. Studies on the research patterns of humanists indicate that they differ from their colleagues in the sciences or social sciences in their approach to information, age and form of material used, and the extent of immediate contact with other researchers. ${ }^{6}$ Documentation of research behaviors of humanists also suggests other characteristics that may be antithetical to using remote collections, e.g., an inclination to work alone, a lack of delegation of their literature searching, and a reliance on browsing as a critical strategy in indentifying the materials of research. ${ }^{7}$

\section{THE UNIVERSITY OF MICHIGAN PROGRAM}

The remote shelving program of the University of Michigan has attempted to ameliorate many of the negative associations with storage. The Buhr Shelving Fa- cility, opened in 1981, has a closed access, compact shelving arrangement and is environmentally controlled for temperature and humidity. Records are created for stored items in the library's online system. Delivery turnaround has typically been within one day, and reading and photocopy facilities are provided on-site. In addition, the storage collection is considered dynamic and volumes can be returned to open stacks if use patterns change.

\section{"To assess the effectiveness of the Michigan program, the library pro- posed to investigate the attitudinal factors surrounding humanistic fac- ulty's acceptance of remotely shelved collections."}

To assess the effectiveness of the Michigan program, the library proposed to investigate the attitudinal factors surrounding humanistic faculty's acceptance of remotely shelved collections. Further, with funding from the Council on Library Resources and the Andrew W. Mellon Foundation, the library also proposed to assess whether attitudes could be improved by creating machine-readable cataloging records for remotely shelved items in relevant disciplines and by providing enhanced bibliographic access through RLIN, the online system of the Research Libraries Group that is used by Michigan for cataloging. Access to RLIN in the academic departments provided an opportunity to analyze a number of interesting issues surrounding the larger questions of immediate physical availability of collections vs. less proximate forms of access, e.g., remote shelving, interlibrary loan or surrogate microformats.

The Humanistic Scholars Project began in January 1983 and ended in December 1985 . The study population was composed of 212 faculty in the departments of English and History and the American Culture program. The study was subsequently extended to include 254 graduate students in the same departments. This 
grant funding enabled the library to create approximately 50,000 machine-readable records in RLIN, supporting the effort to measure effects of increased bibliographic access upon attitudes toward remote shelving.

In addition to the information concerning attitudes toward remote shelving, the project accumulated information that may provide direction for future programs in research libraries. Specifically, the following issues were examined:

1. alterations in humanistic research strategies brought about by changes in library programs and services, e.g., preservation, technology, and remotely housed collections;

2. use patterns of humanities materials in remotely shelved collections;

3. general library use patterns that may suggest policies for future selection of titles for remote shelving and for retrospective record conversion projects;

4. the effect of patron access to online systems on research behaviors and attitudes;

5. training strategies for online systems; and

6. characteristics of humanities researchers that suggest strategies for future library programs.

\section{METHODOLOGY}

The Humanistic Scholars Project included three major efforts: (1) record conversion activities, (2) creation of an instructional/outreach program and its evaluation, and (3) administration of a survey to faculty and graduate students in participating departments.

\section{Record Conversion}

The creation of machine-readable records in RLIN was a significant component of the Humanistic Scholars Project. Concentrating on the subject areas most heavily used by the scholars in our study population, titles from the relevant classifications housed in the Buhr Facility were selected for conversion in RLIN. All titles in storage were also reflected in Geac, the library's online circulation system. As a result, project participants had sophisticated bibliographic access to a select group of relevant titles from storage through RLIN and author/title/call number access to all stored titles via Geac.

Three RLIN terminals were installed in the English and History Departments and in a library classroom for use by project participants. These terminals were available through December 1985.

\section{RLIN Training}

The training component of the project called for individual and group sessions with faculty members and graduate students to demonstrate RLIN's general capabilities and its possible applications in the humanities, including online access to materials at Buhr. During the planning phase, a package of instructional materials was designed that was appropriate to this unique audience and consistent with the hypotheses of the grant. Through direct mailings, posted announcements, newsletters, and general user education activities conducted by the Graduate Library Reference staff, faculty and student participation was encouraged. Finally, evaluation forms were devised to monitor the effectiveness of both the trainers and the instructional materials.

The group reflected a wide range of experience with computers. While many of those trained were complete novices, at least ten percent of the faculty who were trained were owners of microcomputers and thus came to RLIN with some understanding of both the advantages and limitations of this technology. The project employed a graduated set of training materials that moved from the very basic to the sophisticated with the explanations and illustrations drawn from the humanities wherever possible.

Comments from the faculty training evaluation forms indicated a generally positive response to the program and to RLIN as a research tool, although the statements also reflected perceptions of RLIN's shortcomings. Sample comments from these evaluations that reflect the range of reactions include the following:

A convenient way to check bibliographic citations and footnotes in proofreading my publications. On the occasions when I do need to find current works in fields outside of my specialty, I 
anticipate it will be very useful especially by using combined topic headings-something I couldn't do in a regular catalog.

I found the system useful for confirming things I knew or partly knew, but not useful for discovery.

Useful not only in research but in the organization of new courses.

My lack of use is mainly because I have a fairly good command of the sources I most regularly use. The database has not revealed much that is new to me. Perhaps when the terminals are more ubiquitous and the database is larger, I will have more reason to use it with regularity.

Advanced training sessions were offered to the faculty in Winter 1984. In addition, a newsletter was produced to foster awareness of and continued participation in the project.

\section{Survey Design}

During the summer of 1983, the project staff worked simultaneously on the design of the first questionnaire and on the training program, both of which were to be administered in the Fall 1983 academic term. The purpose of the first questionnaire was to measure faculty attitudes and use patterns prior to the intervention of the project.

The questionnaire included three components: a section on current library use, a section to gather demographic information, and a series of attitudinal statements. During the Fall 1983 term, 212 questionnaires were mailed to faculty members in English, History, and American Culture, of which 119 were returned, a response rate of 56 percent. Eighty-eight faculty members subsequently participated in the RLIN training sessions. The project was later expanded to include graduate students in these three academic programs.

When the availability of RLIN ended, the faculty and students were again surveyed. The second questionnaire duplicated the majority of the initial questions but also included questions regarding library services initiated in the intervening years (e.g., public access to the Geac circulation system) that might have affected behavior and attitudes. In December 1985, 195 questionnaires were mailed to the faculty with a response rate of 41 percent.

\section{SURVEY RESULTS \\ Population}

At the outset, a few generalizations about the sample population can be offered based on the data from the completed questionnaires. The faculty respondents to the first questionnaire were predominantly male senior faculty at the rank of full professor, over 40 , tenured, and with the University for 10 years or more. Forty-four percent were in the English Department, 31 percent in History and 25 percent in the American Culture program (more detailed information about the samples, reflecting respondents to the first questionnaire, is shown in appendix A). No significant differences in population characteristics were detected in the sample populations responding to the first and second administrations of the questionnaire.

Both the first and second questionnaires included a series of behavioral questions regarding the respondents' use of the library and its materials and services, as well as attitudinal questions about library storage and technology. Unless otherwise noted, the analysis of the research behavior questions reported below is based upon faculty responses to the first questionnaire only, while the attitudinal responses are compared across the two administrations of the questionnaire. "Humanities scholars involved in
the project were heavy library users;
the modal response ( 57.6 percent)
was weekly use of the central human-
ities and social science library."

\section{BEHAVIORAL MEASURES Library and Computerized Access}

The two surveys provided a wealth of information about research behaviors and library use. In general, it was clear that the humanities scholars involved in the project were heavy library users; the modal response (57.6 percent) was weekly use of the central humanities and social science 
library. Although no significant difference by rank was found, assistant and associate professors were more likely to report daily use of the library (22.2 percent and 30.8 percent respectively) than their full professor colleagues (14.3 percent). Higherranked faculty were more likely to report having checked out large numbers of volumes, yet there was also a negative relationship within the senior ranks between faculty age and the number of items checked out.

Respondents typically reported that they "very often" or "often" work alone (96 percent), gather their own bibliographic citations (78 precent) and retrieve their own materials (87 percent). In general, there was modest use reported by all categories of faculty for library service points (e.g., catalog information, government documents center, reference desk). A majority of respondents indicated that they "never" used online databases or online utilities such as RLIN or OCLC in the library. The data did reveal a significant inverse relationship between age and the use of automation; younger faculty (aged 20 to 40 years) were significantly more likely than their older counterparts to use both online databases $\left(\chi^{2}=34.67\right.$, $\mathrm{p}<.01)$ and RLIN/OCLC $\left(\chi^{2}=24.84\right.$, $\mathrm{p}<.05)$.

\section{Use of Remote Collections}

The survey prompted the faculty to describe their use of remote collections (i.e., interlibrary loan or the Buhr Shelving Facility) as well as reasons for not using these services. For both interlibrary loan and retrieval from the Buhr facility, the majority of respondents indicated that these services were not used because the services were not needed. The questions related to the Buhr facility also give some indication of the perceived effectiveness of the remote shelving program. As noted above, the most common reason given for not using the Buhr facility was that items held there were not needed, followed in order of frequency by "retrieval takes too long," " too difficult to determine what is in storage," and "unaware of library storage collection." A similar response pattern was elicited in the second questionnaire, although significantly fewer respondents indicated that retrieval of Buhr volumes takes too long (5.1 percent in 1985 as compared with 20.2 percent in 1983).

The most extensive use of the Buhr Shelving Facility was concentrated among associate professors. This rank was also the most likely to report that the retrieval process took too long and that it was difficult to determine which items were shelved at Buhr. Although only a few faculty members reported that they were unaware of Buhr (4.3 percent), those choosing this response were concentrated in the more junior ranks.

\section{Bibliographic Sources and Computer Use}

The survey population was asked how frequently they used particular sources of bibliographic information. The most frequently cited bibliographic sources for which some regular use was indicated (i.e., excluding response "never" and "rarely") were the card catalog (97 percent) and citations in books and articles (96 percent). Other commonly cited means of identifying relevant materials were indexes and abstracts (74 percent), browsing the open stacks ( 83 percent), subject bibliographies (86 percent), and book reviews (90 percent). The least used sources were computerized databases and librarians.

Age and rank did not prove to be good predictors of use of particular tools, but those at the lower ranks tended to use a wider array of sources more intensively than their senior colleagues. Another suggestive finding was that female faculty were more likely than males to report reliance upon colleagues, conferences, and librarians as sources of bibliographic information. Gender also proved to be related significantly to computer use; more women (30.6 percent) than men (16.2 percent) reported use of both mainframe and microcomputing systems $(\mathrm{p}<.05)$.

\section{RLIN Use}

Those individuals who responded to the second questionnaire were asked about their use of RLIN following training. Of the 78 individuals responding, 48 indicated that they were trained to use RLIN and 31 respondents actually used RLIN subsequent to training. The two most 
commonly cited reasons for nonuse of RLIN were that they "did not believe RLIN would benefit their work" (49 percent) or that they "preferred to use the card catalog" (51 percent).

The forty-eight faculty who did use RLIN were asked what system characteristics they found most desirable. The fact that they could obtain information about collections at other universities (67 percent), the ability to compile a quick bibliography (44 percent), and the ability to search by key words (40 percent) were most frequently cited as desirable attributes. Interestingly, only two individuals indicated that they liked RLIN because of the ability to search the library's serials records, all of which are contained within the database.

The two major reasons checked to indicate why respondents did not like RLIN were that they used it so infrequently that they forgot the commands or that it did not contain sufficient records for items in the Buhr Shelving Facility.

Finally, the 78 faculty respondents to the second questionnaire were asked to select from a list of twelve possible effects those that best indicated how their involvement in the Humanistic Scholars Project had impacted their work. Respondents could select more than one effect. In general, the most frequent response was that there was no impact on work or behavior, although those respondents who had used RLIN were significantly more likely to report that the Humanistic Scholars Program had an impact on their work (F statistic $=33.226, \mathrm{p}<.01)$.

\section{ATTITUDINAL MEASURES}

The questionnaire included thirty-one attitudinal statements that formed three distinct scales: attitude toward remote shelving (storage), attitude toward technology, and attitude toward the Library. For each attitudinal statement, respondents were asked to indicate the extent of their agreement along a five-point continuum ranging from "strongly disagree" to "strongly agree." Items were randomly distributed in the attitude portion of the questionnaire and their directionality was varied to minimize response bias. Factor analysis was performed to ensure the reliability of the three scales and the results showed acceptable levels of correlation among the scaled items. The three scales and the individual statements are listed in appendix B.

Analysis of the attitudinal portion of the questionnaire focused on the relationship of the responses to the demographic and behavioral factors described above (except where noted the following analysis focuses on responses to the first administration of the questionnaire). Means were calculated based upon the assumption that respondents viewed the five points on the scales as equidistant and distributed about a neutral point of 3.0. The analysis showed that overall the most positive attitudes were recorded on the "Library" scale (3.86), followed respectively by the scales for "technology" (3.76), and "remote shelving" (3.19). The mean score for each of the scales was above the neutral point, indicating overall favorable attitudes toward each of the three factors.

\section{Remote Shelving}

Thirteen attitudinal questions were combined to form a single scale measuring degrees of acceptance or rejection of remote shelving (storage). Age and rank did not prove to be related significantly to storage attitudes, but there was a tendency for older and more senior faculty to hold more positive attitudes toward the concept of remote shelving ( $\mathrm{t}$-value $=$ $2.0754, p<.05)$. Not surprisingly, there was a significant inverse relationship $(r=$ -.39) between stated preference for browsing at the shelves and attitudes toward storage.

Those stating in the behavioral section of the questionnaire that retrieval from Buhr was either "too hard" or "too slow" scored significantly lower on the remote shelving attitude scale than those choosing other responses $(p<.01)$. Those not needing Buhr items were likely to have more positive attitudes toward remote shelving.

The introduction of library technology (RLIN) does not appear to have had an effect upon either reported use of or attitudes toward remote shelving-that is, 
"The introduction of library technology (RLIN) does not appear to have had an effect upon either reported use of or attitudes toward remote shelving."

the availability of this enhanced access tool did not significantly alter faculty attitudes toward storage.

Interestingly, there did not appear to be a relationship between those faculty who indicated two or more reasons for infrequent use of the Buhr storage collection and their attitude toward storage. More specifically, the assumption that those with a larger number of negative perceptions of the Buhr service (as reflected in two or three reasons for nonuse of the Buhr storage collection) might evidence more negative attitudes toward remote shelving in general was not substantiated. A significant negative relationship ( $t-$ value $=-2.9357, \mathrm{p}<.01$ ) was found, however, between those listing two or more reasons for not using Buhr and their overall attitude toward the library. One possible interpretation is that those with a number of reasons for not using Buhr tended to generalize their concern toward the library rather than restricting their negative attitudes toward remote shelving alone.

\section{Technology Attitudes}

Technology attitudes were related to the use of computing resources. There was a significant positive relationship between use of more than one computing resource and positive attitude toward technology in general $(\mathrm{t}$-value $=3.0873, \mathrm{p}<.01)$. Rank and age also proved to be significantly related to technology attitude. Older and more senior ranked faculty were more likely to express negative attitudes toward technology. Other suggestive findings include the fact that faculty with overall positive feelings about technology were more likely than others to work collaboratively and to rely upon peers for information. Not surprisingly, it is this group of faculty who reported higher use of RLIN.

\section{Library Attitudes}

Faculty giving overall positive responses on library attitude statements were significantly more likely to be older and more advanced in rank ( $\mathrm{t}$-value $=$ $3.2940, \mathrm{p}<.01$ ). This group indicated they were likely to have others gather citations for them, but subsequently came to retrieve their own materials from the library. These faculty members with positive attitudes toward the library also showed low use of technology, suggesting an inverse relationship between these two factors ( $t$ value $=3.7604, \mathrm{p}<.01$ )

\section{Attitude Change}

Responses were analyzed for those faculty completing both the first and second questionnaires $(\mathrm{N}=55)$ to measure attitudinal change in the two intervening years. Little change was noted in attitudes toward remote shelving or technology. A significant positive change, however, was noted in attitude toward the library $(p<.01)$. While logically one might assume this change was an outgrowth of a positive experience with RLIN, subsequent analysis showed that RLIN users scored lower than nonusers on the library attitude scale. A more likely explanation for the overall improved attitude toward the library is that faculty members appreciated the initiatives taken by staff regardless of whether they took advantage of the opportunity to use RLIN.

\section{DISCUSSION AND CONCLUSIONS}

What conclusions can be drawn from these complex findings? First, the characteristics of the population suggest some changes in research behavior that are likely to emerge in the coming decade as a significant number of senior faculty become less active or retire. The cohort of associate professors (or those roughly 31-40 years of age) uses the library most intensively and draws upon remotely housed collections most frequently. It is also this group that seems more inclined to try new methods of accessing bibliographic information such as library automation might afford.

A second generalization suggested by the project results is that a technological 
intervention for attenuating attitudes toward remote shelving may have been ineffective given generally low levels of familiarity with computerized systems and the prevailing bias against technology at the time. As one faculty member commented, behaviors may change when terminals are more ubiquitous. At the time of the survey, however, interactions with computers were infrequent and some humanist faculty were wary. Further, because positive attitudes toward the library were associated with negative attitudes toward technology, we may have been trying to improve perceptions of library services such as remote shelving using an unattractive medium. Interestingly, those who were RLIN users actually had more negative attitudes toward storage than their nonuser colleagues.

One confounding aspect of the RLIN system that occurred during the project was the reconfiguration of the RLIN database, no longer allowing a user to isolate easily the records of a single institution. One could argue that the lack of the desired impact of RLIN on remote shelving attitudes was related to the inability to search readily (and browse) University of Michigan records. It was also clear from faculty responses that RLIN was perceived to be less than user friendly and this could have had an effect on participant attitudes. Yet during the project the Library also brought up a publicly accessible and easy-to-use local circulation system, Geac, and input into that database records for all items housed at Buhr. The second questionnaire included questions about use of Geac, yet no change in attitudes toward remote shelving was associated with Geac use.

Our findings suggest that Michigan's remote shelving facility, although not universally accepted by faculty, has been successful. Overall attitude scores were above the neutral point, and responses to individual statements about the preservation role of remote shelving reflected a basic understanding of the rationale and imperative of remotely shelved collections. Perceptions of retrieval time noticeably improved between the two surveys as well. Although RLIN may not have ameliorated negative attitudes, an increased use of Buhr was reported on the second questionnaire without attitudes becoming more negative. If nothing else, the program may have increased awareness of the Buhr Facility and methods of access.

An indirect outcome of the Humanistic Scholars Project was an increase in attention to and interaction with faculty. Not surprisingly, attitudes toward the library improved during the course of the project. Also, those who had others gather citations and perform other front-end library work for them were more positive about the library. These findings reflect two principles libraries might consider in developing programs for faculty. First, meaningful interactions and efforts to become involved in research strategies may have a halo effect on overall faculty support of libraries. Secondly, efforts to remove obstacles from library use or streamline faculty-library interactions may also be advantageous.

There are interesting questions that are yet unresolved concerning the immediate availability of materials. During 1986, the library implemented a document delivery program for faculty. Humanities scholars have proved to be among the heaviest users. Will this service and its potential for making a remote location transparent for the user have an impact on acceptance of remote shelving? As libraries develop more extensive shared resources programs, what will be the faculty reaction? Our findings suggest that faculty may reject such alternatives in principle but still become heavier and more effective users of remotely held resources. In the long run this outcome of making faculty more effective researchers may result in improving attitudes. In the short run, however, it is perhaps too much to ask that faculty not only use a remotely housed collection but profess to like it as well.

Library technology will increase dramatically in the coming years-online catalogs, expert systems, textual datafiles. It is as yet unclear how various segments of the faculty will respond to these developments. Our experiences during this program have provided no definitive answers to what the future holds. Yet, our finding that involvement with this program improved overall attitudes toward the li- 
brary, despite specific concerns about such issues as remote shelving and convenience of access, suggests a need for greater interaction between faculty and library staff. It would appear that creating avenues for involvement and dialogue fosters a generally positive climate conducive to finding mutually acceptable solutions to library/research problems. Both li- brarians and faculty involved with the Humanistic Scholars Project claimed to gain a greater awareness and appreciation of the problems facing their counterparts. It is anticipated that this enhanced understanding will result in the provision of better library service offered to a more supportive campus community.

\section{REFERENCES AND NOTES}

1. William E. Lane, "The Treatment of Books According to the Amount of Their Use," Library Journal 28:9 (July 1903); and Charles W. Eliot, "The Division of a Library into Books in Use and Books Not in Use, with Different Storage Methods for the Two Classes of Books," Library Journal 27:51-56 (July 1902).

2. Ralph E. Ellsworth, The Economics of Book Storage in College and University Libraries (Metuchen, N.J.: Scarecrow, 1969). Other pertinent articles on storage of library materials include the following: John J. Boll, Shelf Browsing, Open Access and Storage Capacity in Research Libraries. Occasional Papers, No. 169 (Arlington, Va.: Educational Resources Information Center (ERIC), ED 260721 1985); Gary S. Lawrence, "A Cost Model for Storage and Weeding Programs," College \& Research Libraries 42:139-47 (March 1981); Richard A. Stayner, "Economic Characteristics of the Library Storage Problem," Library Quarterly 53:313-27 (July 1983).

3. George Piternick, Book Storage in Academic Libraries. A Report Submitted to the Council on Library Resources (Arlington, Va.: Educational Resources Information Center (ERIC), ED 112 835, 1974).

4. Association of Research Libraries, Remote Storage, Systems and Procedures Exchange Center (SPEC) Kit 39 (Washington, D.C.: Association of Research Libraries, 1977).

5. Herman H. Fussler and Julian L. Simon, Patterns in the Use of Books in Large Research Libraries (Chicago: University of Chicago Press, 1969).

6. Sue Stone, "Progress in Documentation-Humanities Scholars: Information Needs and Uses," Journal of Documentation 38, no. 4:292-313 (1982).

7. K.J. Weintraub, "The Humanistic Scholar and the Library," Library Quarterly 50, no.1:26 (1980).

8. Additional monographic titles in English language and literature were converted on other grant funds relevant to the Humanistic Scholars Project. Nearly all of the Library's serials records were converted using Title IIC funds. Of the 2,050,000 titles in the University Library collections, 275,000 titles (13 percent of the collection) were retrospectively converted. A total of 850,000 titles, or 40 percent of the collection's bibliographic records were in machine-readable form and thus available via RLIN at the initiation of the project. In addition, RLIN records were available through the Library's publicly accessible circulation system, Geac, as were all Buhr titles.

\section{APPENDIX A. PROFILE OF SAMPLE POPULATION}

SAMPLE DISTRIBUTION BY SEX

\begin{tabular}{lrr}
\hline \hline Category & Number & Percent \\
\hline Female & 19 & 15.9 \\
Male & 98 & 82.4 \\
Missing Data & 2 & 1.7 \\
Total & 119 & 100.0 \\
\hline
\end{tabular}

SAMPLE DISTRIBUTION BY RANK

\begin{tabular}{lrr}
\hline \hline Category & Number & Percent \\
\hline Lecturer & 5 & 4.2 \\
Assistant Professor & 18 & 15.1 \\
Associate Professor & 26 & 21.9 \\
Professor & 70 & 58.8 \\
$\quad$ Total & 119 & 100.0 \\
\hline
\end{tabular}


SAMPLE DISTRIBUTION BY AGE

\begin{tabular}{lrr}
\hline \hline Category & Number & Percent \\
\hline Less than 30 years & 4 & 3.4 \\
$31-40$ & 25 & 21.0 \\
$41-50$ & 38 & 31.9 \\
$51-70$ & $\frac{52}{43.7}$ \\
$\quad$ Total & 119 & 100.0 \\
\hline
\end{tabular}

\section{APPENDIX B: ATTITUDE STATEMENTS CLUSTERED BY SCALE}

\section{Storage}

1. Microforms are an acceptable medium, given the necessity of preserving deteriorating books.

2. Library books in poor condition should be protected in a restricted area.

3. Finding relevant materials for research is hindered by the inability to browse shelves in library storage collections.

4. Lack of current use is a reasonable criterion for storing library materials.

5. The age of library materials is a reasonable criterion for removing materials to a restricted-access location.

6. Library materials should be immediately accessible to be useful for research.

7. Storage of portions of the library's collections is a realistic solution to constraints of available space.

8. Borrowing books from non-UM libraries (i.e., interlibrary loan) is an acceptable method of obtaining materials for scholarly activities.

9. Storage of the library's collections undermines scholarship and research.

10. Microforms are a better solution to space constraints than storing library materials in closed-access stacks.

11. Placing library materials in storage does not significantly reduce a researcher's use of these items.

12. Microformats are an acceptable substitute for printed materials.

13. Geographic dispersion of library materials on campus presents obstacles for users.

\section{The Library}

1. Library staff are a helpful source of information about library services and policies.

2. Card catalog entries provide too little information about library materials.

3. Library staff are an essential source of bibliographic information.

4. The Library presents obstacles for research and scholarship.

5. The Library provides critical support for research on campus.

6. Card catalogs are frustrating to use because of their complexity.

\section{Technology}

1. Scholarly use of computers will have a positive effect on the academic environment.

2. Computers are too impersonal to be effective.

3. The emphasis on computers in universities will have a negative effect on the quality of education.

4. Computers dehumanize scholarly activity.

5. The use of computers to share information with others will benefit scholarly communication.

6. Computers can significantly improve the efficiency of libraries.

7. Word processors reduce creativity in scholarly writing.

8. Computers will help scholars use libraries more effectively.

9. The need for complex technical skills makes effective use of computers by scholars problematic.

10. Library computer systems cannot accommodate individual strategies for conducting research.

11. Computers will have a positive effect on patterns of scholarly communication.

12. Electronic publishing (e.g., the creation, distribution and access of publications by computer) will diminish the quality of scholarly publications.

NOTE: Response choices for all statements were provided on a five point scale ranging from "strongly disagree" to "strongly agree." 